\title{
Interference and negative priming from ignored distractors: The role of selection difficulty
}

\author{
ELAINE FOX \\ University College Dublin, Dublin, Ireland
}

\begin{abstract}
The relation between distractor interference and negative priming from identical distractors was examined in two experiments. Subjects responded to a target letter, which was indicated by an adjacent bar marker, and attempted to ignore a distracting letter. On prime trials, distracting letters were either compatible or incompatible with the target, allowing for a measure of interference. On subsequent probe trials, previously ignored distractors were sometimes presented as targets, allowing for a measure of negative priming. Reducing the spatial separation between targets and distractors on the prime trial increased the magnitude of interference and negative priming, but these effects appeared to be independent of each other (Experiment 1). In Experiment 2, the prime target location was precued on some trials, but not on others. Precuing attenuated the magnitude of interference, but not that of negative priming effects. This pattern indicates that measures of negative priming and measures of distractor interference on the immediately preceding trial are independent. The results are discussed in terms of a selective inhibition model of selective attention.
\end{abstract}

The visual environment presents the observer with numerous problems of selection. At any one time, there may be multiple objects present; some are relevant to the current behavior and need to be responded to, and some are irrelevant and need to be ignored. The locus of this selection has been a contentious issue for experimental psychologists. For example, Broadbent (1958) argued that selection occurs early in information processing, prior to stimulus identification. Thus, according to Broadbent, little (if any) semantic analysis of ignored stimuli takes place. In contrast, other theorists (e.g., Deutsch \& Deutsch, 1963) have argued that ignored stimuli are identified and processed prior to selective attention (see Allport, 1993; van der Heijden, 1992, for recent reviews).

Until fairly recently, attention theorists working within "early" as well as "late" selection frameworks have assumed that once selection of relevant objects takes place, processing of ignored objects ceases, and their effects gradually dissipate over time to some baseline level (e.g., van der Heijden, 1981). However, some researchers have suggested that the processing of ignored stimuli as well as selected stimuli may be a critical part of selective attention (e.g., Allport, Tipper, \& Chmiel, 1985; Neill, 1977; Tipper, 1985). The idea is that ignored stimuli are

Some of this research was conducted while the author was with Victoria University of Wellington (VUW) and was partially supported by a grant from the VUW Internal Grants Committee. I would like to thank David Crossan for his help with computer programming. I am particularly grateful to Arthur Kramer, James C. Johnston, Tram Neill, and an anonymous reviewer, whose comments facilitated revision of this manuscript. Address correspondence to E. Fox, now at Department of Psychology, University of Essex, Wivenhoe Park, Colchester CO4 3SQ, U.K. (e-mail: efox@essex.ac.uk). actively suppressed and do not passively fade back to a baseline level of activation. The empirical evidence for this idea comes primarily from the phenomenon of negative priming (Tipper, 1985), which is the demonstration that people are slower and/or less accurate in responding to stimuli that are related to recently ignored stimuli. For example, if the task is to name the colors of various words, the response "red" to the word blue printed in red will be slower when it follows the word red printed in green than when it follows the word yellow printed in green (Dalrymple-Alford \& Budayr, 1966; Neill, 1977). It seems that the response to the ignored dimension of the stimulus (i.e., word meaning) is suppressed and hence delays the same response on the following trial. Subsequent research had indicated that similar effects are found across a wide range of selective attention tasks: People are generally slower in responding to an item, or a semantic associate of an item, that they have just ignored (see Fox, 1994b; Neill, Valdes, \& Terry, in press, for comprehensive reviews). Thus, the inhibition of the processing of irrelevant information may well be an important mechanism underlying selective attention.

A traditional index of the semantic analysis of irrelevant information is the amount of interference between target and distractor objects appearing in the same array. This is usually inferred from response times (RTs) that are longer for response-incompatible trials than for responsecompatible trials. An additional index is provided by negative priming, which is a comparison of trials in which the prime distractor becomes the target on a subsequent probe display. Reaction times when prime distractors become probe targets are usually slower than those when the probe target is unrelated to the previous (prime) display. An important theoretical issue concerns the nature of the relationship between these two mea- 
sures of the semantic processing of irrelevant stimuli. There are three logical possibilities. First, it may be that inhibition of distractor processing is responsive to increased interference in the visual environment (see Neill et al., in press, for discussion). This implies that a major function of inhibition (as reflected by negative priming) may be to prevent irrelevant information from taking control of behavior and actions. If this is the case, a strong positive relation would be expected between interference and negative priming, so that increased interference is associated with increased negative priming, and vice versa. There is evidence available to support this proposition. For example, Neill and Lissner (1988) found that in a letter-matching task, conditions that induced greater levels of interference also produced a greater magnitude of negative priming. Further evidence comes from a recent demonstration of increased negative priming when stimulus-response mappings were incompatible (Neill, Valdes, \& Terry, 1992). In a target-localization task, one group of subjects pressed the rightmost key if a target appeared in the rightmost location of a display, or the leftmost key if the target appeared in the leftmost position. For another group of subjects, these response assignments were reversed, so that they pressed the rightmost key to indicate the leftmost position, and so on. It was found that incompatible responses produced substantially more negative priming $(52 \mathrm{msec})$ than did compatible responses $(16 \mathrm{msec})$. Since incompatible stimulusresponse mappings are associated with higher levels of Stroop-like interference (e.g., Fox, 1992; McClain, 1983), this pattern of results suggests that conditions associated with increased interference are also associated with increased negative priming.

A second logical possibility is that interference and negative priming may be inversely related. The rationale for this is that inhibition of distractors may render them less available to produce interference, and vice versa (e.g., Neumann \& DeSchepper, 1992). Thus, inhibited distractors should produce less interference, but more negative priming. There is some evidence for this pattern of results in individual-difference studies. In particular, Anthony Beech and his colleagues (e.g., Beech, Baylis, Smithson, \& Claridge, 1989; Beech \& Claridge, 1987; Beech, Powell, McWilliam, \& Claridge, 1989) have shown that subjects with high-schizotypal personality traits are more susceptible to interference from irrelevant distractors and also show less negative priming from the same distractors. In contrast, individuals with lowschizotypal symptoms show less interference, but more negative priming from irrelevant distractors. However, little evidence for this pattern of results has been found in within-subjects designs; it does not seem to be the case that conditions that induce more negative priming also produce less interference, or vice versa, within subjects (e.g., Beech, Agar, \& Baylis, 1989; Fox, in press, 1994a; Tipper, Weaver, Kirkpatrick, \& Lewis, 1991).

A final possibility is that interference and negative priming are independent processes that are not consistently related to each other in either a positive or a neg- ative direction. The bulk of the evidence seems to support this conclusion. First, irrelevant distractors produce roughly equivalent negative priming under conditions in which the same distractors produce no Stroop-like interference, as well as under conditions in which significant Stroop-like interference is observed (Driver \& Tipper, 1989; Fox, in press). Conversely, Beech, Agar, and Baylis (1989) found differences in the magnitude of negative priming across different types of stimuli (color words vs. pseudocolor words, with the same initial letters; e.g., groit for green), but the magnitude of interference remained constant across the different stimuli. Finally, it has been found that although pattern masking reduces negative priming, it has little impact on interference in target-localization (Tipper et al., 1991) and letter-identification tasks (Fox, 1994a; Neill et al., in press). These results suggest a dissociation between measures of interference and measures of negative priming.

This brief review suggests that the relation between negative priming and interference is by no means clear. In within-subjects studies, there is either a positive relation (Neill \& Lissner, 1988; Neill et al., 1992) or no relation (Beech et al., 1989; Driver \& Tipper, 1989; Fox, in press, 1994a; Tipper et al., 1991). A primary aim of the present study was to attempt to clarify the relation between interference and negative priming in a standard selective attention task. The task was an adaptation of Eriksen and Eriksen's (1974) flanker task, since interference (Eriksen \& Eriksen, 1974; Miller, 1991) and negative priming (Fox, in press, 1994a; Tipper, McQueen, \& Brehaut, 1988) have both been established in this paradigm.

\section{The Flanker Paradigm}

The flanker task (Eriksen \& Eriksen, 1974) is a technique that has been widely used to demonstrate that responses to targets (letters or words) are influenced by spatially separate (and presumably unattended) distracting stimuli. It is difficult to completely eliminate this effect, which has been interpreted as evidence for the automatic semantic analysis of unattended stimuli. For example, Miller (1991) required subjects to categorize a centrally presented letter and to ignore irrelevant flanking letters. It was found that the identities of the flanking letters produced response-compatibility effects, even when they were $5^{\circ}$ of visual angle away from the centrally attended area. These results indicate that unattended stimuli are automatically processed at an abstract level.

On the other hand, however, several researchers have suggested that distractor interference can be reduced by modifications in the structure of the display elements, even when targets and distractors are very close together. For example, Kramer and Jacobson (1991) found that distractor interference could be substantially reduced when distractors were embedded in a separate object from the target, even when the distractor was just $.25^{\circ}$ of visual angle from the target. Other studies have shown that the magnitude of distractor interference $d e$ creases as the spatial separation between targets and dis- 
tractors increases (e.g., Eriksen \& Eriksen, 1974; Miller, 1991). This can be interpreted either as evidence for weaker activation of distant distractors (e.g., Kahneman \& Henik, 1981) or as an indication that distant distractors are more easily rejected as nontargets (Allport, 1989; Driver \& Tipper, 1989). Whatever the correct interpretation of this effect, its robustness provides an opportunity to investigate the impact of increasing interference with decreasing target-distractor separation on the magnitude of negative priming from the same distractors. If a function of the inhibitory process's giving rise to negative priming is to prevent irrelevant information from taking control of actions, there should be an increase in negative priming when distractors are close to the target (producing more interference) and a decrease when distractors are far from the target (producing less interference).

The aim of the current investigation was to investigate whether various experimental manipulations would influence measures of distractor interference and negative priming in the same or in opposite directions. More specifically, in Experiment 1, subjects were presented with a letter-identification task in which target-distractor separation on the prime trial had been manipulated. In Experiment 2 , they were presented with a similar task under conditions in which the location of the prime target letter was either precued or uncued.

\section{EXPERIMENT 1}

In Experiment 1, interference and negative priming from ignored distractors was investigated in a letteridentification task previously described by Tipper et al. (1988). Subjects were required to identify target letters, which were indicated by an adjacent bar marker, while ignoring a distractor letter (e.g., -A B) on prime as well as probe displays. The distracting letters' proximity to the target was systematically manipulated on the prime display in a within-subjects design. On the prime trial, the target and distractor letters were equidistant from fixation at a center-to-center separation of $.97^{\circ}$ (near), $1.7^{\circ}$ (medium), or $2.6^{\circ}$ ( far) of visual angle. Thus, the targets and distractors always fell on retinal locations of equivalent acuity. Brief exposure durations of all displays $(50 \mathrm{msec})$ precluded the possibility of any useful eye movements directed toward the location of the target letter. On the probe display, the target-distractor separation was always $.97^{\circ}$ of visual angle.

\section{Method}

Design. Each trial consisted of a prime and a probe display; every display contained two letters. As in Tipper et al. (1988), the interference effect was assessed by comparing a condition in which the target (specified by an adjacent bar marker) and distractor letters had the same identity (response compatible: e.g., -B B) with a condition in which they had different identities (response incompatible: e.g., -B A). Interference would be indicated by longer RTs in the latter condition.
The priming effects of the ignored distractor were assessed in the subsequent probe trial. The probe stimulus always contained target and distractor letters with different identities. There were three priming conditions. There were probe trials following sameidentity prime trials (e.g., D D- followed by -C B); this condition was necessary given the design, but was of no theoretical interest and thus was not included in the analysis. In the control condition, the probe trials following different-identity prime trials contained target and distractor letters that were different from those in the prime display (e.g., -A C followed by D B-). Finally, in the ignored repetition condition, the distractor in the prime display and the target in the subsequent probe display had the same identity (e.g., -B A followed by $\mathrm{C} \mathrm{A-}$ ).

The experiment contained two within-subjects factors for the prime and the probe displays. For the prime display, the withinsubjects factors were separation (near, medium, far) and interference (response compatible vs. response incompatible). For the probe display, the within-subjects factors were separation (on the previous prime display: near, medium, far) and negative priming (control vs. ignored repetition).

Apparatus and Stimuli. Stimulus presentation and data collection were controlled by an IBM-compatible microcomputer running MEL software (Schneider, 1988). Target and distractor stimuli were the capital letters A, B, C, or D, each subtending approximately $.51^{\circ}$ of vertical and $.25^{\circ}$ of horizontal visual angle at a viewing distance of about $45 \mathrm{~cm}$. The two letters in the prime display were always presented side by side, and a bar marker appeared on the outside of the letter to indicate the target (-A A or A A-). The letters in the probe display were always presented one above the other, and the bar marker appeared to the left of the target letter. This configuration avoided any possible confound of spatial priming.

Procedure. The subjects were informed that they would be dealing with four letters: A, B, C, and D. These letters were printed in black tape on the D, F, J, and K keys, respectively, of a standard computer keyboard. The subjects were encouraged to respond to the letter with the bar marker beside it as quickly as possible by pressing the appropriate key, while trying not to make mistakes. It was emphasized that they should not move their eyes during the trials. Following the practice trials, the subjects were asked whether they had found it difficult not to move their eyes, and they were given the option of repeating the practice trials. None of the subjects took up this option, and all said that they had had no difficulty remaining fixated most of the time. The subjects used the index and middle fingers of each hand to make their responses. A trial was considered correct only if both the prime and the probe displays received correct responses.

The sequence of events within a trial was as follows. A fixation point $(+)$ was presented at the center of the screen for $500 \mathrm{msec}$. This display was replaced by the prime display, which was presented for $50 \mathrm{msec}$. This was followed by a blank screen until a response was made. Following the response, there was a blank screen for $350 \mathrm{msec}$, and then the probe display was presented for $50 \mathrm{msec}$. This was followed by a blank screen until a response was made. There was an intertrial interval of $2 \mathrm{sec}$.

The experimental session consisted of 30 practice trials ( 15 response compatible, 15 response incompatible, all probe trials were control) followed by 360 experimental trials. One hundred eighty of the prime displays contained a distractor with the same identity as the target (response compatible); one third (i.e., 60) of these trials were presented at each of the target - distractor separations. One hundred eighty prime trials contained a different-identity distractor (response incompatible), again with 60 at each targetdistractor separation. There were 30 control and 30 ignored repetition trials at each level of target-distractor separation. The RTs to the 180 probe trials following response-compatible prime trials 
were not analyzed. Thus, each subject completed 390 trials ( 30 practice, 360 experimental) in a single session, which lasted about $35 \mathrm{~min}$.

Subjects. Twenty students ( 12 females, 8 males) from University College Dublin participated in the study. The subjects ranged in age from 18 to 45 years, and all reported normal or correctedto-normal vision. They were not paid for their participation in the experiment.

\section{Results}

Two subjects (both male) made errors on more than $30 \%$ of the trials, so their data were not included in the analysis. The data from the remaining 18 subjects ( $12 \mathrm{fe-}$ males, 6 males, with an age range of 18-29 years) were subjected to statistical analyses.

Prime display. Trials containing errors, or those with RTs less than $150 \mathrm{msec}$ or greater than $1,500 \mathrm{msec}$ on the prime display, were excluded from the data analysis. The means of the median correct RTs and percent errors are presented in Table 1 . The median correct RTs were cast into a 3 (separation: near, medium, far) $\times 2$ (interference: response compatible, response incompatible) analysis of variance (ANOVA) with subjects as the random factor. Separation $[F(2,34)=7.44, p<.01]$ and interference $[F(1,17)=53.4, p<.001]$ showed significant main effects, and there was a significant interaction between them $[F(2,34)=12.8, p<.001]$. Further analyses revealed that the interference effect was significant at the near $[t(17)=-9.15, p<.001]$ and the medium $[t(17)=-6.36, p<.001]$ separations, but not at the far separation. Paired $t$ tests confirmed that the magnitude of interference at the far separation was significantly less than that observed at either the medium separation $[t(17)=4.1, p<.001]$ or the near separation $[t(17)=$ $4.1, p<.001]$. There was no difference in the magnitude of interference observed for near and medium separations $[t(11)<1]$.

A similar ANOVA was conducted on the arcsine transformed error scores. This analysis revealed a significant main effect for interference $[F(1,17)=10.4, p<.01]$; the subjects made more errors $(8 \%)$ in response-incompatible trials than they did in response-compatible trials $(5 \%)$. There was no main effect of separation, but the interfer-

Table 1

Means of Median Reaction Times (in Milliseconds) and Percent Errors (PE) as a Function of Trial Type and Distractor Proximity in Experiment 1

\begin{tabular}{|c|c|c|c|c|c|c|}
\hline & \multicolumn{6}{|c|}{ Target-Distractor Separation } \\
\hline & \multicolumn{2}{|c|}{ Near } & \multicolumn{2}{|c|}{ Medium } & \multicolumn{2}{|c|}{ Far } \\
\hline & $M$ & $\mathrm{PE}$ & $M$ & $\mathrm{PE}$ & $M$ & $\mathrm{PE}$ \\
\hline \multicolumn{7}{|c|}{ Prime Display } \\
\hline Response compatible & 404 & 5 & 403 & 3 & 436 & 7 \\
\hline Response incompatible & 462 & 9 & 453 & 8 & 451 & 7 \\
\hline Interference & +58 & & +50 & & +15 & \\
\hline \multicolumn{7}{|c|}{ Probe Display } \\
\hline Control & 417 & 8 & 430 & 6 & 428 & 7 \\
\hline Ignored repetition & $4 \$ 5$ & 16 & 448 & 16 & 437 & 16 \\
\hline Priming & -38 & & -18 & & -9 & \\
\hline
\end{tabular}

ence $\times$ separation interaction was significant $[F(2,34)=$ $3.7, p<.05]$. The errors and RTs followed the same pattern, and there was no suggestion of a speed-accuracy tradeoff.

Probe display. Trials with errors on either the prime display or the probe display were excluded from the data analysis. Trials with RTs less than $150 \mathrm{msec}$ or greater than 1,500 msec were also excluded. The means of the median correct RTs for the control and ignored repetition probe displays are presented in Table 1 . The median correct RTs were cast into a 3 (separation on the previous prime trial: near, medium, far) $\times 2$ (negative priming: control, ignored repetition) ANOVA with subjects as a random factor. There was no effect of separation on the previous trial, but there was an overall negative priming effect of $22 \mathrm{msec}[F(1,17)=11.3, p<.01]$. Of more interest, the interaction between (prime trial) separation and negative priming was also significant $[F(2,34)=$ $4.3, p<.05]$. Further analysis revealed that the magnitude of negative priming was significant when the prime trial separation had been near $[t(17)=-3.9, p<.001]$ or medium $[t(17)=-2.5, p<.05]$. The negative priming effect when the prime trial separation had been far was not significant. Paired $t$ tests comparing the magnitude of negative priming following each of the different (prime trial) separations revealed that negative priming following the near separation was significantly larger than that following either the medium $[t(17)-3.0, p<.01]$ or the $\operatorname{far}[t(17)=-2.5, p<.05]$ separation. There was no difference in the magnitude of interference following the medium and the far prime trial separations $[t(17)<1]$.

A similar ANOVA on the arcsine transformed error scores revealed a significant main effect for negative priming $[F(1,17)=65.2, p<.001]$; the subjects made fewer errors on control trials $(7 \%)$ than they did on ignored repetition trials $(16 \%)$. No other effect was significant.

\section{Discussion}

The results of this experiment were in line with the experimental predictions. As in previous research, the magnitude of interference from irrelevant distractors increased with decreasing separation between the distractor and the target (e.g., Miller, 1991). Of more interest, however, was the finding that negative priming from ignored distractors also increased in magnitude with decreasing separation from the target. Valdes (cited by Neill et al., in press) also presented target and distractor letters at different degrees of "near" (unspecified) and "far" separations in a letter-identification task. Distracting letters close to the target produced $44 \mathrm{msec}$ of interference and $31 \mathrm{msec}$ of negative priming, and distracting letters far from the target produced $24 \mathrm{msec}$ of interference and just $7 \mathrm{msec}$ of negative priming.

Thus, it seems that when targets and distractors are close together, measures of interference as well as negative priming increase, but they decrease when distractors are farther away from the target. These results indicate that inhibition may indeed be reactive, when applied 
according to the amount of interference actually encountered (Neill et al., in press). Against this, however, the greatest change in the interference effects in the present experiment occurred between the medium- and the farseparation conditions. In contrast, the greatest change in negative priming occurred between the near- and the medium-separation conditions. Thus, the mechanisms underlying the two effects may well be independent. This issue will be returned to in the General Discussion.

The present results indicate that the spatial proximity of distracting stimuli affects inhibitory processes, as reflected by increased negative priming, as well as interference effects. One interpretation of these results is simply that distant distractors are less likely to be identified and therefore produce less interference and less negative priming than do near distractors (Kahneman \& Chajczyk, 1983; Kahneman \& Henik, 1981). Alternatively, it may be the case that "distant distractors produce less interference not because they fail to be identified but because they are readily dismissed as nontargets. By contrast, near distractors are strong candidates for the target because of their locus and hence influence response processes" (Driver \& Tipper, 1989, p. 305). The present results cannot distinguish between these alternatives.

A clearer test to distinguish between these hypotheses is to present the same task under conditions in which selection of the target is very easy. To illustrate, if reduced interference with increasing target-distractor separation is due to less activation of the distant distractors (e.g., Kahneman \& Henik, 1981), then neither interference nor negative priming would be expected from distant distractors if the target location was validly precued. Several studies have shown that precuing target location does indeed reduce or eliminate interference from response-incompatible distractors (Paquet \& Lortie, 1990; Yantis \& Johnston, 1990). However, a series of experiments reported by Fox (in press), demonstrated that although precuing target location does indeed reduce the magnitude of interference effects, the magnitude of negative priming does not decrease in precued relative to uncued trials. Thus, to infer that distractors that produce less interference undergo less semantic processing is clearly erroneous (Driver \& Tipper, 1989). A potential problem with the results of Fox (in press), however, is that precuing was manipulated either between subjects or within subjects in separate blocks. This design leaves open the possibility that the subjects may have utilized some conscious strategic process, since the presence of informative or noninformative cues was always predictable from trial to trial. The aim of Experiment 2, therefore, was to attempt to replicate the previous results under conditions in which the precuing of target location was fully randomized within subjects. Thus, from trial to trial, subjects could not predict whether they would receive a valid cue to prime target location, or simply a noninformative temporal warning.

\section{EXPERIMENT 2}

\section{Method}

Apparatus and Design. The apparatus and stimuli were the same as those in Experiment 1, except that the center-to-center separation between targets and distractors was always $1.9^{\circ}$ of visual angle on the horizontal (prime display) and the vertical (probe display) axis. As in Experiment 1, the target letter (A, B, C, or D) in both displays was indicated by means of an adjacent bar marker. On some trials, the location of the prime target was indicated by a 150 -msec presentation of a single asterisk in the location of the forthcoming target. On uncued trials, two asterisks were presented for $150 \mathrm{msec}$ - one in the location of the forthcoming target and one in the location of the forthcoming distractor. Hence, the same temporal warning was given in both conditions, but only the single cue provided information about the location of the target. No cues were presented on probe trials.

Thus, the experiment contained two within-subjects factors. For the prime display, the within-subjects factors were precuing (cue, no cue) and interference (response compatible, response incompatible). For the probe display, the factors were precuing on the previous prime display (cue, no cue) and negative priming (control, ignored repetition).

Procedure. The instructions given to the subjects were the same as those given in Experiment 1. Once again, they were required to respond to the identity of the target letter by pressing one of four keys-A, B, C, or D-which were indicated in black on the D, F, $\mathrm{J}$, and $\mathrm{K}$ keys, respectively.

The sequence of events within a trial was as follows. A fixation point $(+)$ was presented at the center of the computer screen for $500 \mathrm{msec}$. Then, either a single asterisk was presented in the location of the impending target (cued condition), or two asterisks were presented--one in the location of the impending target and one in the location of the impending distractor (uncued condition). The duration of the cue or temporal warning was $150 \mathrm{msec}$. This display disappeared and was immediately replaced by the prime display, which was presented for $50 \mathrm{msec}$. This was followed by a blank screen until a response was made. After the response, there was a blank screen for $350 \mathrm{msec}$, and then the probe display was presented for $50 \mathrm{msec}$. This was followed by a blank screen until a response was made, and then there was a 2 -sec pause until the beginning of the next trial.

The experimental session consisted of 48 practice trials; half were in the cued condition, and half were in the uncued condition. Half of each of these trials (12) contained response-compatible distractors, and half (12) contained response-incompatible distractors. All of the practice probe trials were control trials. Following a brief pause, the subjects began the experimental session, which consisted of 384 trials. Half of these (192) were precued. Ninetysix of these precued prime trials contained the same-identity distractor (response compatible) and 96 contained a different-identity distractor (response incompatible). There were 48 trials in the control and ignored repetition conditions, respectively. The RTs to the 96 probe trials following response-compatible prime trials were not analyzed. The breakdown of the 192 uncued trials was the same. The 384 trials were presented in a different randomized order to all the subjects. Thus, each subject completed 432 trials (48 practice, 384 experimental). There was a short break of about 1 min after 192 of the experimental trials. The entire session lasted about $35 \mathrm{~min}$.

Subjects. Twelve students ( 8 females, 4 males) were recruited from an undergraduate course at Victoria University of Wellington. None of the subjects had participated in Experiment 1. The subjects ranged in age from 19 to 25 years. All reported normal or corrected-to-normal vision, and were paid NZ $\$ 5.00$ for their participation in the experiment. 


\section{Results}

Prime display. Trials containing errors, or those with RTs less than $150 \mathrm{msec}$ or greater than $1,500 \mathrm{msec}$ on the prime display, were excluded from the data analysis. The means of the median correct RTs and percent errors are presented in Table 2 . The median RTs were cast into a 2 (precuing: cue, no cue) $\times 2$ (interference: response compatible, response incompatible) repeated measures ANOVA with subjects as a random factor. The analysis revealed that responses to the prime target when its location had been precued were faster than those in the uncued condition [392 vs. $417 \mathrm{msec}$, respectively; $F(1,11)=$ $10.1, p<.01]$. There was also a significant main effect for interference; RTs in the response-incompatible trials were significantly longer than those in the responsecompatible trials [415 vs. $394 \mathrm{msec}$, respectively; $F(1,11)=39.4, p<.001]$. However, these effects were modified by a significant precuing $X$ interference interaction $[F(1,11)=39.0, p<.001]$. Further analysis revealed that the interference effect of $8 \mathrm{msec}$ in the cued condition did not reach significance $[F(1,11)=3.9, p<$ $.07]$, but the interference effect of $34 \mathrm{msec}$ in the uncued condition was significant $[F(1,11)=87.6, p<.001]$.

A similar analysis of the arcsine transformed error scores revealed that the results generally paralleled the RT results. The main effect for precuing did not reach significance, but there was a significant main effect for interference $[F(1,11)=25.1, p<.001]$; the subjects made more errors $(6 \%)$ on response-incompatible trials than they did on response-compatible trials (3.5\%). As with the RT results, there was a significant precuing $\times$ interference interaction $[F(1,11)=6.1, p<.05]$. Further analysis revealed that the apparent increase in errors on response-incompatible precued trials did not quite reach significance $[F(1,11)=4.3, p<.06]$. However, the subjects did make a significantly greater number of errors on response-incompatible trials than they did on responsecompatible trials when the prime target was uncued $[F(1,11)=63.5, p<.001]$.

Probe display. Trials containing errors on either the prime or the probe displays were excluded from the data analysis. The means of the median correct RTs for the control and ignored repetition probe displays are shown

Table 2

Means of Median Correct Reaction Times (in Milliseconds) and Percent Errors (PE) as a Function of Trial Type and Prime Target Precuing in Experiment 2

\begin{tabular}{lcccc}
\hline & \multicolumn{3}{c}{ Cuing Condition } \\
\cline { 2 - 3 } & \multicolumn{2}{c}{ Cue } & & No Cue \\
\cline { 2 - 5 } & $M$ & PE & & PE \\
\cline { 2 - 5 } & Prime Display & & & \\
Response compatible & 388 & 3 & 400 & 4 \\
Response incompatible & 396 & 5 & 434 & 7 \\
Interference & +8 & & +34 & \\
& Probe Display & & & \\
Control & 404 & 3 & 409 & 4 \\
Ignored repetition & 441 & 4 & 423 & 5 \\
Priming & -37 & & -14 & \\
\hline
\end{tabular}

in Table 2. These were cast into a 2 (precuing on the previous trial: cue, no cue) $\times 2$ (negative priming: control, ignored repetition) repeated measures ANOVA with subjects as a random factor. There was no main effect for precuing, but the overall negative priming effect of $25 \mathrm{msec}$ was significant $[F(1,11)=88.8, p<.001]$. However, there was also a significant precuing $\times$ negative priming interaction $[F(1,11)=30.1, p<.001]$. Further analysis revealed that negative priming was significant when the prime target was precued $[37 \mathrm{msec} ; F(1,11)=$ $104.0, p<.001]$ and when there was no precue [14 msec; $F(1,11)=18.7, p<.01]$. The significant interaction shows that the magnitude of negative priming when the prime target was precued was significantly greater than that in the uncued condition.

A similar analysis was conducted on the arcsine transformed error scores; this revealed no significant main effects and no significant precuing $\times$ negative priming interaction. There was no evidence of a speed-accuracy tradeoff.

\section{Discussion}

The pattern of results observed in Experiment 2, in which precued and uncued prime trials were randomized within subjects, replicates previous results with a similar task (Fox, in press, Experiments 1 and 2). First, there was a reliable reduction in the magnitude of interference from response-incompatible distractors when the location of the prime target was reliably precued. Second, there was a reliable increase in the magnitude of negative priming from ignored distractors when the prime target location was precued. This result is inconsistent with the conclusion that precuing target location prevents the semantic analysis of spatially separate distracting information (see Paquet \& Lortie, 1990; Yantis \& Johnston, 1990).

It should be noted, however, that there is a possible artifact in the precuing manipulation used in Experiment 2 . Since the precue was a single asterisk presented in a location that was the same as that for the forthcoming target, the precue may have inadvertently produced forward masking of the target. The abrupt onset of the distractor (in the cued condition) from a previously empty field may also have had the undesirable effect of attracting attention to the distractor location. Thus, the cue used here may not have worked very well to keep spatial attention on the target position. Fox (in press, Experiment 3) conducted an experiment to eliminate the possibility of forward masking by the cuing manipulation by using precues that did not appear in the exact position of the stimuli. Under these conditions, the magnitude of interference was again reduced by a precue, but the magnitude of negative priming remained constant across precued as well as uncued conditions. Thus, the increase in negative priming observed in the present Experiment 2 may have been an artifact of the particular precuing manipulation used. The important point, however, is that this artifact does not detract from the central point at issue. Experiment 2 demonstrates that in- 
creases in selection efficiency, as reflected by reduced distractor interference, are not accompanied by a corresponding decrease in the magnitude of negative priming observed. This remains the case even when the possibility of forward masking is removed (Fox, in press, Experiment 3). Thus, reduced distractor interference cannot be attributed to the absence of the semantic processing of ignored objects (see Driver \& Tipper, 1989; Fox, 1994b; Neill et al., in press, for discussion).

It should be noted at this point that the usefulness of negative priming as an index of the semantic processing of irrelevant information has been questioned because of the possibility that the distracting information may be identified after target processing (Gathercole \& Broadbent, 1987). On this view, negative priming tells us little about what happens during selective attention. There are a number of problems with such a delayed distractor identification account of negative priming. Of particular relevance for the current context, there is evidence that the meaning of a stimulus can be perceived even though a subject cannot detect its presence or absence at above-chance levels (Marcel, 1983; see Reingold \& Merikle, 1993, for discussion). When subjects are not consciously aware of a stimulus, positive priming is observed (Allport et al., 1985; Marcel, 1983), which reverses to negative priming when they are aware of the stimulus (Allport et al., 1985; Neill et al., in press). These results indicate that although the implementation of inhibitory processing may be delayed relative to target processing, the identification of irrelevant information probably is not.

\section{GENERAL DISCUSSION}

In the present study, the magnitude of interference and negative priming effects was examined in association with variations in target-distractor separation (Experiment 1) and variations in precuing prime target location (Experiment 2). There were two main results. When tobe-ignored distractors were presented close to the prime target, the magnitude of interference and the subsequent negative priming was greater than that when distractors were moved farther away from the target. On the other hand, precuing prime target location reduced the magnitude of interference effects, but did not decrease the magnitude of subsequent negative priming relative to uncued trials.

\section{Relation Between Prime Interference and Negative Priming}

The main objective of the present research was to investigate the nature of the relation between interference and negative priming from ignored distractors. As was pointed out in the introduction, previous research has shown that the two indices can be either positively related (Neill \& Lissner, 1988; Neill et al., 1992; Valdes, 1993), or not related at all (Allport et al., 1985; Beech, Agar, \& Baylis, 1989; Driver \& Tipper, 1989; Fox, in press; Tipper et al., 1991). The results from the present
Experiment 1 provide some support for a positive relation, since negative priming as well as interference increased in magnitude as distractors and targets were placed closer together (see also Valdes, 1993). However, a close examination of the data suggests that the effects of target-distractor separation on interference and negative priming, although superficially similar, do not appear to be parallel. To illustrate, in Experiment 1, the magnitude of interference decreased most from the mediumto the far-separation conditions. In contrast, negative priming decreased somewhat more from the near- to the medium-separation conditions. This apparent dissociation might be taken as evidence for separate underlying mechanisms. This hypothesis is supported by the results of Experiment 2, which indicated a clear dissociation between measures of interference and negative priming. Precuing prime target location significantly reduced the magnitude of interference, but the magnitude of negative priming did not decrease (see Driver \& Tipper, 1989; Fox, in press, for similar results).

In summary, it seems that improving perceptual segregation between target and distractor objects (e.g., presenting targets and distractors in different colors, precuing the target, etc.) affects interference, but not negative priming (Driver \& Tipper, 1989; Fox, in press), whereas masking or stimulus type affects negative priming, but not interference (Beech, Agar, \& Baylis, 1989; Fox, 1994a; Tipper et al., 1991). Finally, target-distractor separation on the prime trial affects interference and negative priming in the same direction, but apparently does so in an independent fashion (see, e.g., the present Experiment 1).

\section{A Selective Inhibition Model of Selective Attention}

What are the implications of independence between measures of distractor interference and negative priming for our understanding of selective attention? A straightforward explanation of negative priming, based on the notion of selective inhibition (e.g., Neill, 1989; Neumann \& DeSchepper, 1992; Tipper, 1985; Yee, 1991), is as follows: All items in the visual field are identified, processing of target objects is then activated, while further processing of distracting objects is inhibited. This inhibition persists from prime to probe displays, causing the negative priming phenomenon. It is widely assumed that inhibitory processes are engaged in order to accomplish selection of target objects An important function of such an inhibitory mechanism is presumably to allow for uninterrupted behavioral performance by reducing the amount of interference from distracting objects, and/or to prevent unwanted stimuli from entering conscious awareness (see Milliken, Tipper, \& Weaver, 1994; Neill et al., in press). If interference is taken as an index of performance, and negative priming is taken as an index of inhibition, then the two measures should be related in some consistent way. Specifically, the assumption is that negative priming is causally related to interference. However, the empirical data suggest that measures of prime trial interference and measures of 
subsequent negative priming are not consistently related to each other, but appear to be independent (Beech, Agar, \& Baylis, 1989; Driver \& Tipper, 1989; Fox, in press, 1994a; Tipper et al., 1991).

The apparent dissociation between interference and negative priming presents a problem for selective inhibition theory only if it is assumed that inhibition should lead to a reduction of interference on the current trial. An important point to note is that the implementation of inhibition takes some time. First, if distractors are masked shortly after presentation, positive priming is likely to occur, whereas longer exposure of distractors leads to negative priming (Allport et al., 1985; Neill et al., in press; see also Yee, 1991). This suggests that distracting information is initially activated and subsequently suppressed. Second, if subjects are encouraged to respond rapidly to target objects, the nature of priming from ignored items tends to be positive (i.e., facilitatory). However, when accuracy is emphasized and responding is slower, ignored objects are more likely to produce negative priming (Neill \& Westberry, 1987; Neumann \& DeSchepper, 1992). Thus, the implementation of inhibition takes time. A plausible hypothesis is that inhibition might lead to a reduction of interference on subsequent trials, even though it may not be effective in reducing interference on the trial during which the inhibition is applied. Support for this notion comes from the finding that responses when previously ignored distractors as well as targets are repeated are faster than responses when just targets are repeated (Neumann \& DeSchepper, 1991). This is presumably because inhibited distractors are less available to produce interference on the next trial. If inhibition can be implemented faster than usual (e.g., if the prime target is precued), then perhaps inhibition can be applied in time to reduce prime trial interference. This offers a possible explanation for the significant reduction in interference observed in Experiment 2 , when the prime target was precued. Perhaps precuing allowed for an easy distinction between the target and the distractor, thus allowing for a faster implementation of inhibition on the precued trials. The extra time required for target detection in the uncued trials may have prevented the inhibitory mechanism from operating fast enough to reduce interference on the current trial. In the natural environment, irrelevant stimuli are likely to remain irrelevant, and therefore inhibition of processing of such stimuli is likely to improve performance of sequential actions. Since it is only rarely that a response is required to a recently ignored item, the cost associated with distractor inhibition would not be too detrimental to sequential actions.

This view can account for the current results, which show that reducing the proximity between prime target and distractor objects leads to (apparently independent) increases of both interference and negative priming. Distracting items that are very close to a target are more difficult to dismiss as nontargets (Allport, 1989; Driver \& Tipper, 1989), and therefore more interference, is experienced. Distracting items that are very close to the tar- get are stronger competitors for the control of action, and this may lead to the implementation of stronger inhibition of distractor processing. To illustrate, imagine a lioness hunting deer. The target deer might be selected on the basis of some distinguishing feature (e.g., a distinctive color) or because it is somewhat apart from the rest of the herd. In order to smoothly capture the selected deer, the lioness might inhibit further processing of other distracting deer. Animals that are closer to the target deer are more likely to distract the lioness, and, therefore, inhibition applied to the nearby animals would be stronger than that applied to those farther away. The important point for the present purposes is that this inhibition leads to less interference on subsequent actions, but not necessarily on the initial response. This notion is compatible with recent demonstrations that inhibition is determined to a large extent by the behavioral goals of the task (Milliken et al., 1994; Tipper, Weaver, \& Houghton, in press). These studies indicate that only distracting information that is likely to disrupt the current behavioral goal is inhibited. Distracting items that are present in the scene, but do not conflict with the behavioral goal, are unlikely to be inhibited.

\section{Does Negative Priming Reflect a Protective Mechanism?}

An important assumption underlying the current view is that negative priming is determined to a large extent by the presence of potentially conflicting information. A similar view has recently been presented by Moore (1994), who demonstrated that various manipulations on the probe trial determine whether or not negative priming is observed. To illustrate, when no conflicting information (i.e., a distractor) was present on the probe trial, no negative priming was generally observed. Of more interest, when no conflicting information was present on the probe trial, but when this was difficult for the subject to determine, substantial negative priming was observed. Moore concluded that negative priming is associated with a process that is responsible for protecting the system from eliciting a response that is based on incorrect information. The results of the present Experiment 1 support this notion. When prime trial distractors were more likely to elicit a wrong response (i.e., were closer to the target), a greater magnitude of subsequent negative priming was observed. Likewise, Milliken et al. (1994) have also found a small increase in negative priming with increasing difficulty in prime target selection.

The results from Experiment 2, however, are more difficult to reconcile with this account. When distractor interference disappeared altogether (i.e., precued trials), significant negative priming was still observed (see also Allport et al., 1985; Driver \& Tipper, 1989; Fox, in press). If negative priming reflects a protective mechanism, why is it implemented when target selection is very easy and no interference is apparent? Likewise, although there is compelling evidence that probe trial conflict is an important determinant of negative priming (Moore, 1994), other studies have shown significant negative priming 
even though no probe trial conflict was present (e.g., Yee, 1991). Thus, although prime and probe trial conflict can influence the magnitude of negative priming effects, conflict is apparently not a necessary condition, since some studies have shown negative priming even when a protection process would seem to be unnecessary.

\section{Conclusions}

The present results indicate that negative priming effects are independent of interference effects on the immediately preceding trial. More research is required to clarify the implications of this independence for a theoretical understanding of selective attention. It is suggested that selective inhibition theory can account for this dissociation, since inhibition takes some time to be effective. Thus, an inhibitory process may not reduce interference during the trial in which it is applied, but it is effective in allowing uninterrupted sequential actions. Since inhibited objects rarely become target objects in the natural world, the associated cost of inhibition is not overly detrimental to behavior. It is suggested that negative priming is a reflection of a protective (inhibitory) mechanism, which serves to prevent unwanted stimuli from taking control of thoughts or actions (see also Milliken et al., 1994; Moore, 1994; Neill et al., in press). It is acknowledged, however, that the presence of negative priming in the absence of interference (Allport et al., 1985; Driver \& Tipper, 1989; Fox, in press; see also the present Experiment 2) presents some difficulty for an account of negative priming based on the idea of a protective mechanism (see Moore, 1994). It is concluded that the specific conditions that may be associated with the presence or absence of negative priming require further investigation.

\section{REFERENCES}

Allport, D. A. (1989). Visual attention. In M. I. Posner (Ed.), Foundations of cognitive science (pp. 631-682). Cambridge, MA: MIT Press.

AlLPORT, D. A. (1993). Attention and control: Have we been asking the wrong questions? A critical review of 25 years. In D. E. Meyer \& S. Kornblum (Eds.), Attention and performance XIV: A silver jubilee (pp. 183-218). Hillsdale, NJ: Erlbaum.

Allport, D. A., Tipper, S. P., \& Chmiel, N. R. J. (1985). Perceptual integration and postcategorical filtering. In M. I. Posner \& O. S. M. Marin (Eds.), Attention and performance XI (pp. 107-132). Hillsdale, NJ: Erlbaum.

BEECH, A., AgAR, K., \& BAylis, G. C. (1989). Reversing priming while maintaining interference. Bulletin of the Psychonomic Society, 27, 553-555.

Beech, A., Baylis, G. C., Smithson, P., \& Claridge, G. (1989). Individual differences in schizotypy as reflected in measures of cognitive inhibition. British Journal of Clinical Psychology, 28, 117-129.

BEECH, A., \& ClaRIDGE, G. (1987). Individual differences in negative priming: Relations with schizotypal personality traits. British Journal of Psychology, 78, 349-356.

Beech, A., Powell, T., McWilliam, J., \& Claridge, G. (1989). Evidence of reduced "cognitive inhibition" in schizophrenia. British Journal of Clinical Psychology, 28, 110-116.

Broadbent, D. E. (1958). Perception and communication. Oxford, U.K.: Pergamon.

DALRYMPLE-Alford, E. C., \& Budayr, B. (1966). Examination of some aspects of the Stroop color-word test. Perceptual \& Motor Skills, 23, 1211-1214.

Deutsch, J. A., \& Deutsch, D. (1963). Attention: Some theoretical considerations. Psychological Review, 70, 80-90.

DRIVER, J., \& TIPPER, S. P. (1989). On the nonselectivity of selective seeing: Contrasts between interference and priming in selective attention. Journal of Experimental Psychology: Human Perception \& Performance, 15, 304-314.

ERIKSEN, B. A., \& ERIKSEN, C. W. (1974). Effects of noise letters upon the identification of a target letter in a nonsearch task. Perception \& Psychophysics, 16, 143-149.

Fox, E. (1992). Stimulus-response compatibility as a determinant of interference in a Stroop-like task. Bulletin of the Psychonomic Society, 30, 377-380.

Fox, E. (1994a). Masking attenuates negative priming but not interference. Manuscript in preparation.

Fox, E. (1994b). Semantic processing of irrelevant information in selective attention: Evidence from negative priming. Manuscript submitted for publication.

Fox, E. (in press). Precuing target location reduces interference but not negative priming from visual distractors. Quarterly Journal of Experimental Psychology.

GATHERCOLE, S. E., \& BroAdBent, D. E. (1987). Spatial factors in visual attention: Some compensatory effects of location and time of arrival of nontargets. Perception, 16, 433-443.

KahNEMAN, D., \& CHAJCZYK, D. (1983). Tests of the automaticity of reading: Dilution of Stroop effects by color-irrelevant stimuli. Journal of Experimental Psychology: Human Perception \& Performance, 9, 497-509.

Kahneman, D., \& HeniK, A. (1981). Perceptual organization and attention. In M. Kubovy and J. R. Pomerantz (Eds.), Perceptual organization (pp. 181-211). Hillsdale, NJ: Erlbaum.

Kramer, A. F., \& JaCoBson, A. (1991). Perceptual organization and focused attention: The role of objects and proximity in visual processing. Perception \& Psychophysics, 50, 267-284.

MARCEL, A. J. (1983). Conscious and unconscious perception: Experiments on visual masking and word recognition. Cognitive Psychology, 15, 197-237.

MCClaIN, L. (1983). Stimulus-response compatibility affects auditory Stroop interference. Perception \& Psychophysics, 33, 266-270.

MILLER, J. (1991). The flanker compatibility effect as a function of visual angle, attentional focus, visual transients, and perceptual load: A search for boundary conditions. Perception \& Psychophysics, 49 , 270-288.

Milliken, B., Ttrper, S. P., \& Weaver, B. (1994). Negative priming in a spatial localization task: Feature mismatching and distractor inhibition. Journal of Experimental Psychology: Human Perception \& Performance, 20, 624-646.

MOORE, C. M. (1994). Negative priming depends on probe-trial conflict: Where has all the inhibition gone? Perception \& Psychophysics, 56, 133-147.

NEILL, W. T. (1977). Inhibitory and facilitatory processes in selective attention. Journal of Experimental Psychology: Human Perception \& Performance, 3, 444-450.

NeILL, W. T. (1989). Lexical ambiguity and context: An activationsuppression model. In D. S. Gorfein (Ed.), Resolving semantic ambiguity (pp. 63-83). New York: Springer-Verlag.

NeILL, W. T., \& Lissner, L. S. (1988, April). Attention and selective inhibition in alphanumeric matching. Paper presented at the meeting of the Eastern Psychological Association, Buffalo, NY.

Neill, W. T., VAldes, L. A., \& Terry, K. M. (1992, November). Negative priming in target localization. Paper presented at the meeting of the Psychonomic Society, St. Louis, MO.

Neill, W. T., VAldes, L. A., \& TerRy, K. M. (in press). Selective attention and the inhibitory control of cognition. In F. N. Dempster and C. J. Brainerd (Eds.), New perspectives on interference and inhibition in cognition. New York: Academic Press.

NeILl, W. T., \& WeSTbERRY, R. L. (1987). Selective attention and the suppression of cognitive noise. Journal of Experimental Psychology: Learning, Memory, \& Cognition, 13, 327-334.

Neumann, E., \& DeSchepper, B. G. (1991). Costs and benefits of tar- 
get activation and distractor inhibition in selective attention. Journal of Experimental Psychology: Learning, Memory, \& Cognition, 17. $1136-1145$

Neumann, E., \& DeSchepper, B. G. (1992). An inhibition-based fan effect: Evidence for an active suppression mechanism in selective attention. Canadian Journal of Psychology, 46, 1-40.

Paquet, L., \& Lortie, C. (1990). Evidence for early selection: Precuing target location reduces interference from same-category distractors. Perception \& Psychophysics, 48, 382-388.

Reingold, E. M., \& MerikLe, P. M. (1993). Theory and measurement in the study of unconscious processes. In M. Davies \& G. W. Humphreys (Eds.), Consciousness: Psychological and philosophical essays (pp. 40-57). Cambridge, MA: Blackwell.

SCHNEIDER, W. (1988). Micro Experimental Laboratory: An integrated system for IBM PC compatibles. Behavior Research Methods, Instruments, \& Computers, 20, 206-217.

TipPER, S. P. (1985). The negative priming effect: Inhibitory priming by ignored objects. Quarterly Journal of Experimental Psychology, 37A, $571-590$.

TipPer, S. P., MacQueen, G. M., \& Brehaut, J. C. (1988). Negative priming between response modalities: Evidence for the central locus of inhibition in selective attention. Perception \& Psychophysics, 43, 45-52.
Tipper, S. P., Weaver, B., \& Houghton, G. (in press). Behavioural goals determine inhibitory mechanisms of selective attention. Quarterly Journal of Experimental Psychology.

TipPer, S. P., Weaver, B., Kirkpatrick, J., \& Lewis, S. (1991). Inhibitory mechanisms of attention: Locus, stability, and relationship with distractor interference effects. British Journal of Psychology, 82, 507-520.

VALDES, L. A. (1993). The relation of negative priming to interference. Unpublished doctoral dissertation, Adelphi University, Garden City, NY.

VAN DER HEIJDEN, A. H. C. (1981). Short term visual information forgetting. London: Routledge \& Kegan Paul.

VAN DER HeIJDEN, A. H. C. (1992). Selective attention in vision. London: Routledge \& Kegan Paul

YANTIS, S., \& JoHnston, J. C. (1990). On the locus of visual selection: Evidence from focused attention tasks. Journal of Experimental Psychology: Human Perception \& Performance, 16, 135-149.

YEE, P. L. (1991). Semantic inhibition of ignored words during a figure classification task. Quarterly Journal of Experimental Psychology, 43A, 127-153.

(Manuscript received September 13, 1993; revision accepted for publication June 1,1994 .) 